\title{
Continuous, monodisperse silver nanoparticles synthesis using microdroplets as a reactor
}

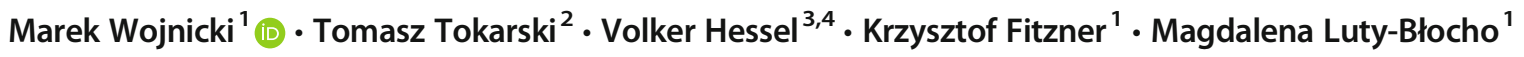

Received: 29 October 2018 /Revised: 17 November 2018 / Accepted: 20 November 2018 / Published online: 26 November 2018

(C) The Author(s) 2018

\begin{abstract}
In this paper precisely controlled silver nanoparticles synthesis method using microdroplets as a reactor was shown. It was confirmed that the application of microdroplets effects in a decrease in particles size and size distribution in comparison with a typical batch reactor from $4.8 \pm 1.3 \mathrm{~nm}$ to $2.5 \pm 0.5 \mathrm{~nm}$. This decrease of particles size distribution is directly related to the fact, that thanks to the application of microdroplets as reactors the mass transfer limits were eliminated.
\end{abstract}

Keywords Droplets · Microreactor · Silver nanoparticles · DMAB

\section{Introduction}

In the last decade, the synthesis of nanomaterials has made a large step forward, due to their potential application in many areas such as catalysis $[1,2]$, medicine [3, 4], optics [5], tissue [6], etc. Nanomaterials have due to their high surface to volume ratio [7] unique properties different from bulk materials such as optical $[8,9]$ and electronically [10] properties. Two methods are at hand for the synthesis of nanomaterials, i.e. physically [11] or chemical [12] based. Efficient nanomaterial syntheses provide particles of defined size, morphology, composition, performance property, etc. However, the choice of synthesis route depends

Marek Wojnicki

marekw@agh.edu.pl

1 Faculty of Non-Ferrous Metals, AGH University of Science and Technology, Al. A. Mickiewicza 30, 30-059 Krakow, Poland

2 Academic Centre for Materials and Nanotechnology, AGH University of Science and Technology, Ave. A. Mickiewicza 30, 30-059 Krakow, Poland

3 Department of Chemical Engineering and Chemistry, Micro Flow Chemistry and Process Chemistry, Technische Universiteit Eindhoven, P.O. Box 513, 5600, MB Eindhoven, The Netherlands

4 School of Chemical Engineering, The University of Adelaide, Adelaide, Australia on a few more factors: equipment cost, production intensity, type of reagents, and complexity of synthesis $[13,14]$. Taking into account these factors, a commonly used protocol is the chemical reduction of metal precursor with reductant in an aqueous media. This chemical reduction can be conducted in batch reactor or in microreactor.

Microfluidic systems allow a particularly good process control. This is most evident from their ability to tune nanoparticle size, and narrow the nanoparticles size distribution $[9,10,15-22]$. The high volumespecific ratio of channel surface area to volume enables ultrafast mixing and fast heat exchange which are of utmost relevance to the seed formation and particle growth. Essentially the same feature, however, also promotes adsorption of the formed nanostructures and such fouling can finally lead to system blocking.

In this paper we like to show the application of sequential flow for silver nanoparticles synthesis. Sequential flow comprises a chain of aqueous droplets in a moving continuous organic medium. Different from segmented flow, the droplets are smaller than the channel diameter and thus do not attach to the channel surface through a very thin liquid capping layer. Brutchey et al. [23] were investigating microfluidic droplet flows of ionic liquids for silver and gold nanoparticles synthesis. They have shown that the AuNPs synthesized in batch were 29\% larger 
than those produced in the microfluidic device. Also Yang et al. [24] were investigating continuous flow process for silver nanoparticles synthesis. In both mentioned above papers, experiments were performed in non-aqua media. What probably makes these technologies more expensive in compere with water base systems.

In our previse paper [25], optimal conditions for droplet formation were investigated. From this study, essential process conditions for the droplet formation were determined like the phase composition and the flow rate.

In this paper, we proposed the application of a chain of droplets as a set of little reactors, which flows within a continuous phase, i.e. the sequential flow. We would like to check if it is possible to decrease the nanoparticles size distribution formed as compared to a typical batch reactor. We hope that the sequential flow might give similar advantages for nanoparticle synthesis as known for the segmented flow. For this a fast reaction reduction of silver(I) ions and a homogeneous process of nucleation and growth in all reaction volume will be needed.

\section{Experimental}

\section{Chemicals}

Silver nanoparticle synthesis was performed using silver(I) nitrate(V) $\left(\mathrm{AgNO}_{3}\right.$, Avantor Materials) as metal precursor. A stock solution containing $\mathrm{Ag}^{+}$ions was prepared as follows: $16.987 \mathrm{~g}$ of $\mathrm{AgNO}_{3}$ was dissolved in $0.1 \mathrm{M}$ of $\mathrm{HClO}_{4}$ (Avantor Performance Materials, P.A) to finally obtain $0.1 \mathrm{~mol} / \mathrm{dm}^{3}$ solution. This solution was stored in brown glass bottle. Borane dimethylamine complex 97\% (DMAB, Fluka) was used as the reducing agent. Heptane (Avantor Materials, P.A), was used as oil phase. Span 80 (delivered by Sigma Aldrich), polyvinyl alcohol (PVA, Sigma Aldrich, $\mathrm{MW}=67,000$ ) and Tween 80 (Avantor Performance Materials, P.A) were used as surface active agents to obtain stable and homogeneous droplets. The last two polymers (PVA and Tween 80) may also act as silver nanoparticle stabilizing agents.

In all experiments concerning the application of the two phase system (in this paper termed as heterogeneous system), the water phase consists of $1 \%$ $\operatorname{PVA}(\mathrm{m} / \mathrm{v}$ ratio) and $1 \%$ of Tween $80(v / v$ ratio). The concentration of $\mathrm{Ag}^{+}$ions was equal to $2 \times 10^{-4} \mathrm{~mol} /$ $\mathrm{dm}^{3}$, and the oil phase consists of heptane and $1 \%$ of Span80 $(v / v)$.
In case of the homogenous system, all reagents were dissolved in water. Since Span 80 is insoluble in water, this polymer was not applied. For this purpose two solutions were prepared. The first one, contains the reductant $\left(20 \mathrm{mmol} / \mathrm{dm}^{3}\right.$ of DMAB), and $1 \%$ PVA $(\mathrm{m} / \mathrm{v})$ and $1 \%$ of Tween $80(v / v)$. The second one, comprising the precursor was prepared by dilution of stock solution of $\mathrm{Ag}^{+}$in water to finally obtain concentration of $\mathrm{Ag}^{+}$ equal to $2 \times 10^{-4} \mathrm{~mol} / \mathrm{dm}^{3}$ and $1 \% \operatorname{PVA}(\mathrm{m} / \mathrm{v})$ and $1 \%$ of Tween $80(v / v)$. Next, those two solutions were mixed in a volume ratio $1: 1$ to obtain AgNPs. This set of experiments is termed as homogeneous system.

\section{Analysis methods}

To obtain low flow pulsation during the silver nanoparticles synthesis, syringe pumps (Injectomat Agilla, Fresenius Kabi) were used.

HR-TEM analysis was performed using the instrument (HR - TEM) - FEI TECNAI TF 20 X-TWIN. For this purpose, one drop of freshly prepared colloidal suspensions was placed on a copper grid covered by a $20-30 \mathrm{~nm}$ amorphous carbon film. Then, the samples were left to dry at room temperature (c.a. $\left.20^{\circ} \mathrm{C}\right)$.

The zeta potential of the obtained material as well as the size and size distribution were determined using the Zetasizer Nano ZS (Malvern Instrument). For this purpose, a standard clear polycarbonate cell with gold electrodes was used.

UV-Vis spectra were registered using the Shimadzu model U-2501 PC. $10 \mathrm{~mm}$ and $2 \mathrm{~mm}$ path length quartz cuvettes were used for the analysis, and base-line corrected against the reference water filled quartz cuvette.

$\mathrm{XRD}$ analysis was performed using an $\mathrm{X}$ - ray diffractometer (Rigaku MiniFlexII). For this purpose, ca. $5 \mathrm{~mL}$ of the obtained colloidal sample was slowly evaporated on the holder surface. Next, the analysis was performed.

\section{Experimental conditions of the silver nanoparticle synthesis and microchannel flow configuration}

The silver nanoparticle synthesis was performed in two different reactor systems which are homogeneous (water) phase in a batch reactor and the microdroplets reactor. The synthesis conditions have been kept identical in all cases, concerning the reductant and precursor concentration, temperature, and the stabilizing agents addition. In that way, it is ensured that all kinetically relevant parameters have been set the same, and thus reaction kinetics are the same. As there is then also no heat transport issue, only mass transport through diffusion can have impact on the particles size and size 
distribution. The process of silver nanoparticle formation in microdroplets was conducted in a microreactor, taking into account the results (flow rate conditions) obtained in our previous study [25], in which it the common sense was followed that increasing mixing rate (up to elimination of diffusion) can lead to the formation of monodisperse particles.

In Fig. 1 the microchannel network with a chain of the formed drops is shown. The channels marked as 1,2, 3 were used to inject the water phase (1, 3 reductant, 2-precursor) into the system, whereas the channels 4 and 5 were used to inject the oil phase.

The flow rate of the oil phase was equal to $5 \mathrm{~mL} / \mathrm{h}$, and the flow rate of the water phase was equal to $1 \mathrm{~mL} / \mathrm{h}$ [25].

It can be seen (Fig. 1), that for the selected flow condition, the formed droplets (water phase) are uniform in size and shape.

It should be underlined that the water droplets das not have a contact with the reactor walls during silver nanoparticles synthesis reaction. This effective avoid the system blocking.

\section{Results and discussion}

In Fig. 2 the colours of the AgNPs solutions obtained under different conditions are shown. There is significant difference in the sample. In case of the photography shown in Fig. 2 A, colloids were obtained in the homogenous system (batch reactor), with both reductant and precursor being dissolved in the water phase. The color of the sample is darker in comparison with the colloid shown in Fig. $2 b$.

Figure $2 \mathrm{~b}$ shows the colloidal silver obtained in the two phase system (microdroplets) with the reductant and precursor in the water phase. The color of the sample is much lighter compared to those shown in Fig. 2a.

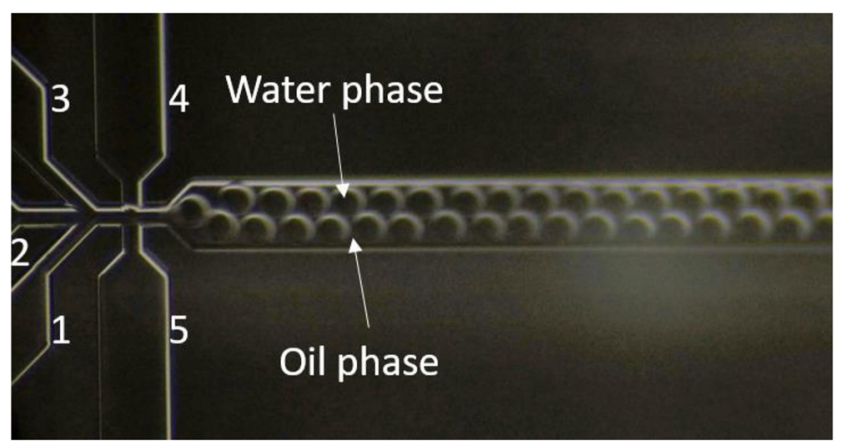

Fig. 1 Water droplet formation in two phase flow. Experimental conditions: oil phase flow rate $2.5 \mathrm{~mL} / \mathrm{h}$ by imputs 4 and 5 . Inputs 1 and 3 were fided by reductant - flow rate $0.25 \mathrm{~mL} / \mathrm{h}$ per chanel and imput 2 was fided by precursor - flow rate $0.5 \mathrm{~mL} / \mathrm{h}$

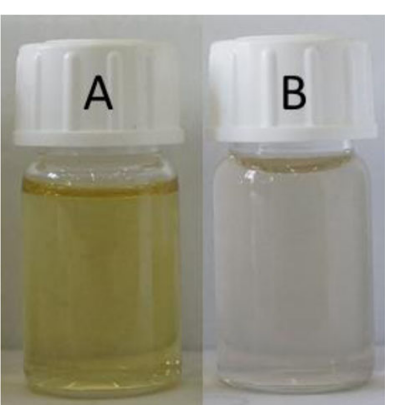

Fig. 2 Photography of the solutions obtained after the synthesis of AgNPs. a batch reactor, $\mathbf{b}$ microflow droplets system

In order to confirm our supposition, the size and size distribution of the AgNPs as well as the zeta potential values were analyzed for the studied cases. The results are given in Table 1. As it can be seen, the particle size obtained in the droplets in the homogenous system (sample B) is significantly lower in comparison to the synthesis conducted in the batch reactor (sample A). Moreover the size distribution of the obtained particles in the microreactor are very narrow and can be assumed as monodisperse.

For all colloids, the values of the zeta potential were registered and are also depicted in Table 1. It was observed, that the zeta potential changes from $-5 \mathrm{mV}$ for the reaction reduction of $\operatorname{Ag}(\mathrm{I})$ ions with $\mathrm{DMAB}$ when being conducted in the homogeneous system in the batch reactor up to $-14.7 \mathrm{mV}$ for reaction when being conducted in microdroplets.

Additionally, after each experiment, the plasmon resonance for the silver nanoparticles was registered, as shown in Fig. 3a and b. Depending on the conditions (batch reactor, droplets system), two different plasmons were obtained with different values of the maximum absorption band. This value change from $390 \mathrm{~nm}$ (Fig. 3b) for the silver nanoparticles obtained in the droplets under mixing control, up to $406 \mathrm{~nm}$ for the particles obtained in the batch reactor (Fig. 3a). It suggests, that the final size of AgNPs in the case of the

Table 1 Particle size and size distribution obtained in batch and microdroplets reactor

\begin{tabular}{lllll}
\hline Fig. 2 & System type & $\begin{array}{l}\text { Average } \\
\text { particles } \\
\text { diameter } *, \mathrm{~nm}\end{array}$ & $\begin{array}{l}\text { Size } \\
\text { deviation, } \\
\mathrm{nm}\end{array}$ & $\begin{array}{l}\text { Zeta } \\
\text { potential, } \\
\mathrm{mV}\end{array}$ \\
\hline $\mathrm{A}$ & Batch reactor & 4.8 & \pm 1.3 & $-5.1 \pm 6.8$ \\
$\mathrm{~B}$ & $\begin{array}{l}\text { Homogenous } \\
\text { system in micro } \\
\text { droplets }\end{array}$ & 2.5 & \pm 0.5 & $-14.7 \pm 5.8$ \\
& & & & \\
& & & & \\
& & &
\end{tabular}

* - distribution calculated taking into account the particles number using DLS method 
Fig. 3 UV-Vis spectrum of AgNPs containing colloids a obtained in the batch reactor $\mathbf{b}$ obtained in micro droplets in the homogeneous system

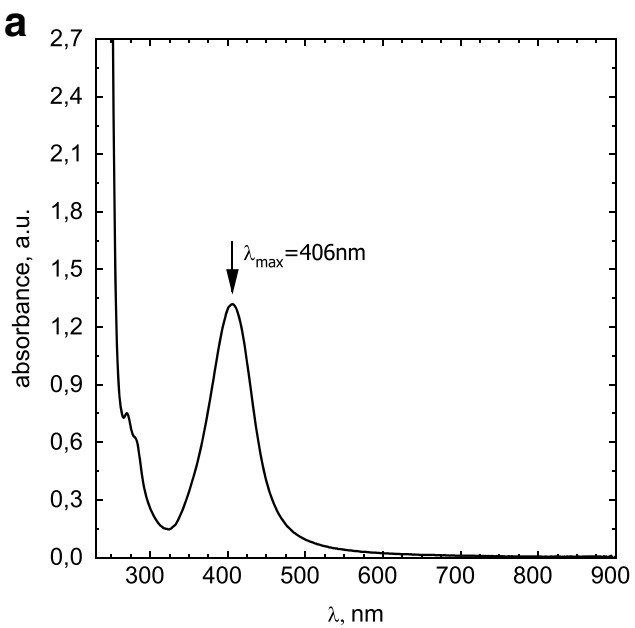

batch reactor should be larger than in the case of the microdroplets, what is in good agreement with DLS analysis (Table 1). Moreover, an additional analysis using HR-TEM were performed to confirm obtained results.

The obtained images are shown in Fig. 4a and Fig. 5a.

HR-TEM image of the sample obtained in the batch reactor is shown in Fig. 4a. In part B of this figure particle size histogram is shown. As it can be seen, there is an excellent agreement between results obtained using DLS and TEM method.

In the case of the AgNPs synthesized in microdroplets, a significant decrease of particle size and size distribution was observed. The crystallography analysis showed only FCC structures (see fig Fig. 5a and $\mathrm{b}$ ). In part $\mathrm{C}$ of this figure particle size histogram is also shown.

Additionally, an XRD analysis was performed for the sample of AgNPs synthesized in microdroplets. For this purpose, about $5 \mathrm{~mL}$ of the solution was

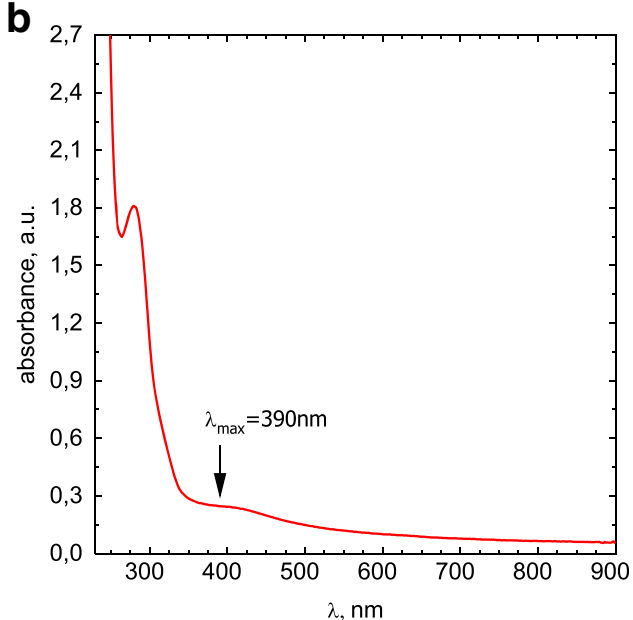

evaporated to obtain a dry sample appropriate for XRD analysis. The result obtained is shown in Fig. 6 $\mathrm{A}$ and $\mathrm{B}$.

Two broad peaks can be observed which are actually composed of two elemental peaks. The significant broadening of those peaks relates to the nanocrystalline structure.

According to the Scherrer equation the single crystal size can be estimated as follows (see eq. (1)).

$d=\frac{K \cdot \lambda}{\beta \cdot \cos \theta}$

where

$\mathrm{d}$ is the mean size of the crystalline, $\mathrm{nm}$

$\mathrm{K}$ is the shape factor, in this case assumed as 0.94 , A.U.

$\lambda$ is the X-ray wavelength, in this case equal to $0.15418, \mathrm{~nm}$

$\beta$ is the fitted peak broadening at half the maximum (FWHM), rad

$\theta \quad$ is the Bragg angle, rad
Fig. 4 a HR-TEM analysis of the sample obtained in the batch reactor, b particle size histogram; the number of particles taken into account is 112
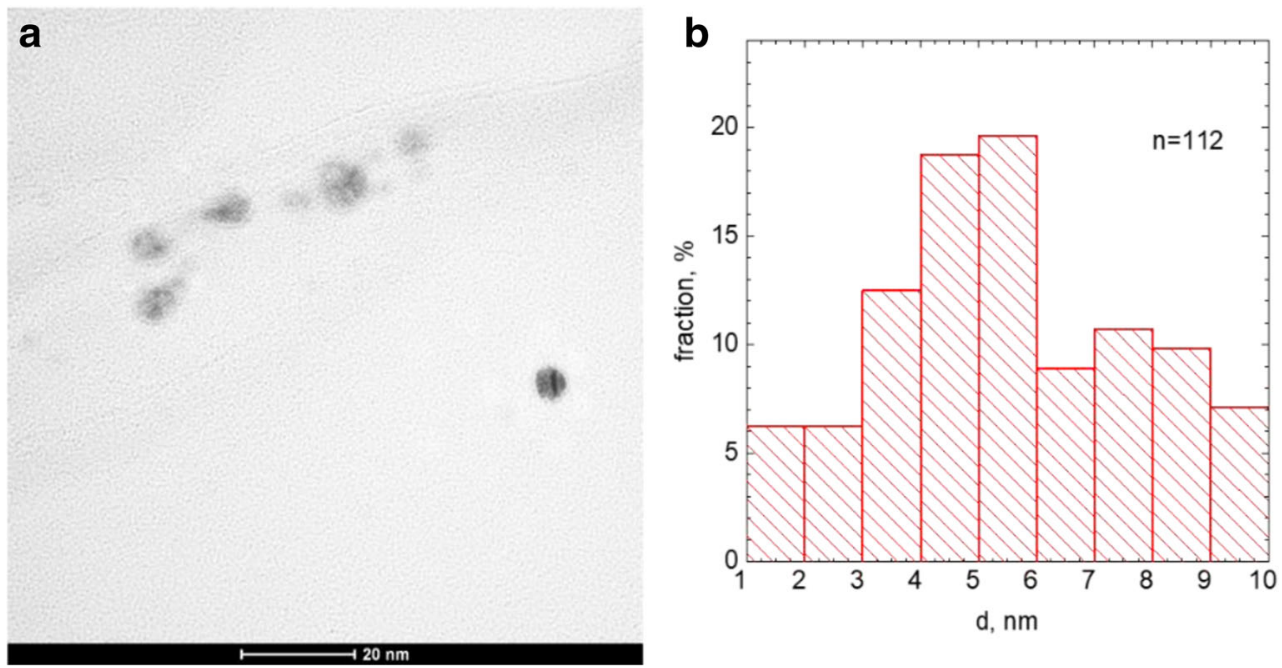

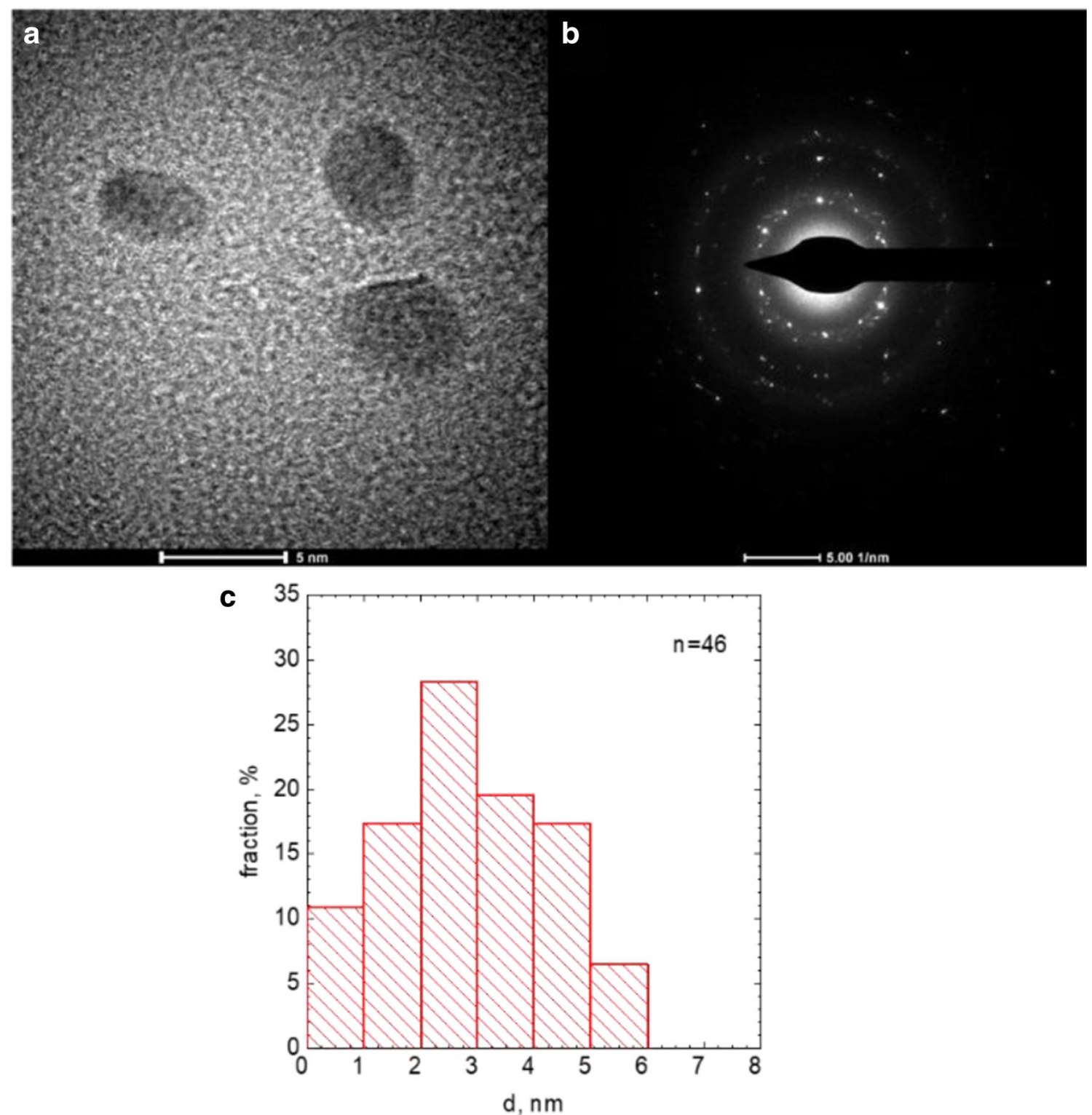

Fig. 5 a HR-TEM analysis of the sample obtained in micro droplets in the homogenous system, $\mathbf{b}$ crystallography structure analysis, $\mathbf{c}$ particle size histogram

In Fig. $6 \mathrm{~B}$ the deconvolution of the selected peak with the highest intensity is shown. It is known that this peak should consist of two elemental subpeaks with maximum at 38.1 for the crystallographic plane $(1,1,1)$ and 44.37 for the crystallographic plane $(2,0,0)$. The numeric analysis reveals that the FWHM equals to 8.6 ${ }^{\circ}$ and $9.3^{\circ}$ respectively. Taking above into account the crystalline mean size was calculated to equal 1.02 and $0.96 \mathrm{~nm}$ respectively. This in turn suggests that there is no shape anisotropy, what is in good agreement with the HR-TEM analysis. Moreover, it confirms the observation of a polycrystalline structure for the obtained nanoparticles.

\section{Summary and conclusion}

In this paper, we have shown, that the polydispersion of nanoparticles is associated with diffusion limit during synthesis. Improvement of mixing during the reaction leads to a decrease of particles size and size distribution. In microdroplets, the gradient of concentration of reagents is eliminated much faster than in a typical batch reactor. Even, microflow reactors are limited since mixing is mainly controlled by diffusion. In the case of microdroplets, interphase friction is a driving force for mixing. It was observed that thanks to the application of microdroplets effective size of nanoparticles were decreased from $4.8 \pm 1.3 \mathrm{~nm}$ to $2.5 \pm 0.5 \mathrm{~nm}$. 
Fig. 6 XRD analysis of AgNPs obtained in micro droplets

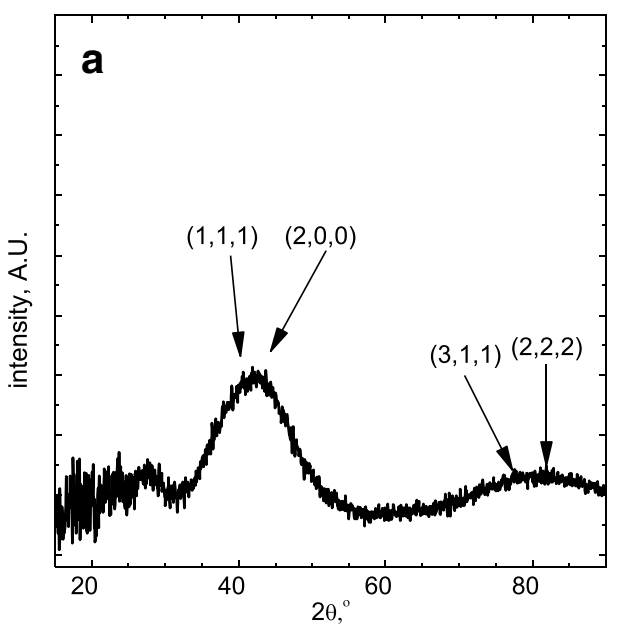

It should be noted, that the application of two-phase flow, allowed to not only control the particles size distribution but also, prevent from nanoparticles deposition at the walls of the microreactor. During our experiments, the whole setups were working continuously $12 \mathrm{~h}$ without visible effects of nanoparticles deposition on the walls of the microreactor.

It is expected that the observed effect can be reproducible regarding the size and uniformity of other metallic nanoparticles such as gold, platinum, palladium or even semiconductors such as $\mathrm{TiO}_{2}$ or $\mathrm{ZnO}$.

Funding This study was funded by the Polish Ministry of Science and Education (grants no IP2014051773 Iuventus Plus).

\section{Compliance with ethical standards}

Conflict of interest The authors declare that they have no conflict of interest.

Open Access This article is distributed under the terms of the Creative Commons Attribution 4.0 International License (http:// creativecommons.org/licenses/by/4.0/), which permits unrestricted use, distribution, and reproduction in any medium, provided you give appropriate credit to the original author(s) and the source, provide a link to the Creative Commons license, and indicate if changes were made.

\section{References}

1. Wojnicki M, Luty-Błocho M, Dobosz I, Grzonka J, Pacławski K, Kurzydłowski K, Fitzner K (2013) Electro-oxidation of glucose in alkaline media on graphene sheets decorated with gold nanoparticles. Mater Sci Appl 4(2):162-169

2. Zhao S, Zhang K, Bai Y, Yang W, Sun C (2006) Glucose oxidase/ colloidal gold nanoparticles immobilized in Nafion film on glassy carbon electrode: direct electron transfer and electrocatalysis. Bioelectrochemistry 69:158-163

3. Kruk T, Szczepanowicz K, Stefańska J, Socha RP, Warszyński P (2015) Synthesis and antimicrobial activity of monodisperse copper nanoparticles. Colloids Surf B Biointerfaces 128:17-22
4. Bednarski M, Dudek M, Knutelska J, Nowiński L, Sapa J, Zygmunt M, Nowak G, Luty-Błocho M, Wojnicki M, Fitzner K, Tęsiorowski M (2015) The influence of the route of administration of gold nanoparticles on their tissue distribution and basic biochemical parameters: in vivo studies. Pharmacol Rep 67(3):405-409

5. Gharibshahi E, Saion E (2012) Influence of dose on particle size and optical properties of colloidal platinum nanoparticles. Int J Mol Sci 13(11):14723-14741

6. Terentyuk GS, Maslyakova GN, Suleymanova LV, Khlebtsov BN, Kogan BY, Akchurin GG, Shantrocha AV, Maksimova IL, Khlebtsov NG, Tuchin VV (2009) Circulation and distribution of gold nanoparticles and induced alterations of tissue morphology at intravenous particle delivery. J Biophotonics 2(5):292-302

7. Kang SW, Lee YW, Park Y, Choi BS, Hong JW, Park KH, Han SW (2013) One-pot synthesis of trimetallic Au@ PdPt core-shell nanoparticles with high catalytic performance. ACS Nano 7(9):7945-7955

8. Knauer A, Schneider S, Möller F, Csáki A, Fritzsche W, Köhler JM (2013) Screening of plasmonic properties of composed metal nanoparticles by combinatorial synthesis in micro-fluid segment sequences. Chem Eng J 227(0):80-89

9. Wojnicki M, Luty-Błocho M, Grzonka J, Pacławski K, Kurzydłowski KJ, Fitzner K (2013) Micro-continuous flow synthesis of gold nanoparticles and integrated deposition on suspended sheets of graphene oxide. Chem Eng J 225(0):597-606

10. Wojnicki M, Luty-Błocho M, Mech K, Grzonka J, Fitzner K, Kurzydowski KJ (2015) Catalytic properties of platinum nanoparticles obtained in a single step simultaneous reduction of Pt(IV) ions and graphene oxide. J Flow Chem 5(1):22-30

11. Iravani S, Korbekandi H, Mirmohammadi SV, Zolfaghari B (2014) Synthesis of silver nanoparticles: chemical, physical and biological methods. Res Pharm Sci 9(6):385-406

12. Nadagouda MN, Varma RS (2007) A greener synthesis of Core (Fe, $\mathrm{Cu}$ )-Shell ( $\mathrm{Au}, \mathrm{Pt}, \mathrm{Pd}$, and $\mathrm{Ag}$ ) nanocrystals using aqueous vitamin C. Cryst Growth Des 7(12):2582-2587

13. Robertson K (2017) Using flow technologies to direct the synthesis and assembly of materials in solution. Chem Cent J 11:4-4

14. Makgwane P, Sinha Ray S (2014) Synthesis of nanomaterials by continuous-flow microfluidics: a review. J Nanosci Nanotechnol 14:1338-1363

15. Yang S-Y, Cheng F-Y, Yeh C-S, Lee G-B (2010) Size-controlled synthesis of gold nanoparticles using a micro-mixing system. Microfluid Nanofluid 8:303-311

16. Shestopalov I, Tice JD, Ismagilov RF (2004) Multi-step synthesis of nanoparticles performed on millisecond time scale in a microfluidic droplet-based system. Lab Chip 4:316-321 
17. Clifford D, El-Gendy A, Lu A, Pestov D, Carpenter E (2014) Room temperature synthesis of highly magnetic cobalt nanoparticles by continuous flow in a microfluidic reactor. J Flow Chem 4(3):148-152

18. Chen-HsunWeng C-C, Huang C-S, Yeh H-Y, Lei G-B (2008) Lee. Synthesis of hexagonal gold nanoparticles using a microfluidic reaction system. J Micromech Microeng 18:1-8

19. Jahn A, Reiner JE, Vreeland WN, DeVoe DL, Locascio LE, Gaitan M (2008) Preparation of nanoparticles by continuous-flow microfluidics. J Nanopart Res 10:925-934

20. Luty-Błocho M, Wojnicki M (2015) Single-step synthesis of onionlike au - Pd - PtNPs nanoparticles using microflow system. J Flow Chem 5(4):197-200

21. Baber R, Mazzei L, Thanh NTK, Gavriilidis A (2017) An engineering approach to synthesis of gold and silver nanoparticles by controlling hydrodynamics and mixing based on a coaxial flow reactor. Nanoscale 9(37):14149-14161

22. Liu G, Ma X, Sun X, Jia Y, Wang T (2018) Controllable synthesis of silver nanoparticles using three-phase flow pulsating mixing microfluidic chip. Adv Mater Sci Eng 2018:1-14

23. Lazarus LL, Riche CT, Marin BC, Gupta M, Malmstadt N, Brutchey RL (2012) Two-phase microfluidic droplet flows of ionic liquids for the synthesis of gold and silver nanoparticles. ACS Appl Mater Interfaces 4(6):3077-3083

24. Lin XZ, Terepka AD, Yang H (2004) Synthesis of silver nanoparticles in a continuous flow tubular microreactor. Nano Lett 4(11): 2227-2232

25. Wojnicki M, Luty-Błocho M, Hessel V, Csapó E, Ungor D, Fitzner K (2018) Micro droplet formation towards continuous nanoparticles synthesis. Micromachines 9(5):1-12 